\title{
Monitoring of Cell Migration and Apoptosis in Cerebellum of Juvenile Masu Salmon Oncorhynchus Masou After Injury
}

\author{
Stukaneva M. E. ${ }^{1}$, Puschina E. V. ${ }^{2}$, Varaksin A. A. ${ }^{2}$, Shukla S. ${ }^{3}$ \\ ${ }^{1}$ Department of Cell Biology and Genetics, Far Eastern Federal University, Vladivostok, Russia \\ ${ }^{2}$ Laboratory of Cytophysiology, A. V. Zhirmunsky Institute of Marine Biology Far Eastern, Branch of Russian Academy of Sciences, \\ Vladivostok, Russia \\ ${ }^{3}$ Prof. Brien Holden Eye Research Centre, L.V. Prasad Eye Institute, Hyderabad, India
}

\section{Email address:}

puschina@mail.ru (pushchina E. V.)

\section{To cite this article:}

Stukaneva M. E., Puschina E. V., Varaksin A. A., Shukla S.. Monitoring of Cell Migration and Apoptosis in Cerebellum of Juvenile Masu Salmon Oncorhynchus Masou After Injury. American Journal of BioScience. Special Issue: Adult and Reparative Neurogenesis: Actual Questions. Vol. 3, No. 2-3, 2015, pp. 19-27. doi: 10.11648/j.ajbio.s.2015030203.13

\begin{abstract}
Apoptosis in various cerebellar zones of juvenile masu salmon Oncorhynchus masou after mechanical injury was investigated by TUNEL-labeling. In the brain of 4 month-old juveniles of $O$. masou growth in different parts of cerebellum and proliferative activity in secondary matrix zones of cerebellum were continued. TUNEL-labeling was observed in the injured cerebellum 2 days after damage. The induction of proliferative activity in different matrix zones: granular eminences, dorsal part of molecular layer and surface layers of corpus cerebellum were noted. The proliferative activity in regional neurogenic niches is increased after injury of cerebellum. The maximal number of apoptotic elements in the cerebellum was observed in zones of the radial cell migration. We suggest that in damaged cerebellum both adult born cells and cells formed as a result of the reparative neurogenesis can be eliminated during radial migration as result of natural morphogenetic processes. Patterns of tangential and radial migration of cells were observed near the area of injury. The highest rate of apoptosis index was detected in the dorsal matrix zone corresponding to the zone of secondary neurogenesis. This testifies to the elimination of the newly formed cells at the stage of their localization in the matrix zones. The intensity of the apoptotic response varies in different areas of $O$. masou cerebellum.
\end{abstract}

Keywords: Apoptosis, Injury of Cerebellum, Dorsal Matrix Zone, Adult Neurogenesis, Oncorhynchus masou, Tangential and Radial Migration of Cells, Reparative Neurogenesis

\section{Introduction}

Growth and differentiation of some integrative centers of brain in lower vertebrates during the post-embryonic stage of development has been reported [1]. It happens as a result of adult neurogenesis. Different species of fish examined thus far also generates new neurons constitutively, both in large numbers and in many regions of the adult CNS. After brain injury the natural processes of post-embryonic growth and proliferation processes are added to reparative neurogenesis. They are provided by the formation of additional centers of cell proliferation in the main areas of secondary neurogenesis, parenchymal cells, and activation of neurogenic niches [2]. During ontogenesis the speed of cell proliferation in the different part of nervous system is not same. The highest level of cell proliferation is observed during embryogenesis and early post-embryonic development; and in adults it is significantly decreased [3].

Adult neurogenesis in fishes is closely related to the ability to regenerate neural tissue after injury $[4,5]$. However, it remains unknown how the process of reparative neurogenesis correlates with cell proliferation and apoptosis. It was shown that the nervous tissue of fish is restored within a few weeks after injury [6]. The high regenerative potential of these animals is determined by the sequence of some morphogenetic processes, one of them is apoptosis. This process is a programmed cell death, regulated by genes and plays a major role in some normal and pathological conditions. Fish are an interesting object for investigation of neurogenic processes, because in the CNS of fish the morphogenetic processes are rather strong and continue during all life. On other hand, fish are evolutionary ancient group with defined high concentration of undifferentiated 
elements in the matrix zones of brain and in nervous tissue's parenchyma [7]. This is especially evident for phylogenetically ancient groups of fish, which include salmoniformes (Salmonidae). Comparative analysis has suggested that adult neurogenesis is a primitive vertebrate trait $[1,2,5]$. It is likely that the availability of all the cellular and molecular regulatory mechanisms necessary for the generation of new neurons in the intact CNS has greatly facilitated the repurposing of the cellular machinery for neuronal regeneration with only slight adaptive changes. A broad understanding of the biology of adult neurogenesis and neuronal regeneration will also facilitate the analysis of the selective pressures that have caused the loss of the regenerative potential during the evolution of mammals.

The purpose of this work was to investigate the apoptotic response and changes in the morphological structure 2 days after experimental injury of cerebellum in juvenile Masu salmon Oncorhynchus masou.

\section{Materials and Methods}

\subsection{Animals}

We used 10 juveniles of masu salmon Oncorhynchus masou (age: 4 months) with average body length 5-6.5 cm and average body weight 14-15.7 $\mathrm{g}$ from Ryasan local breeding hatchery. Masu salmon were maintained at $16-18^{\circ} \mathrm{C}$ with a 14/10 h light-dark cycle and a 1 times/day diet.

All animal manipulations were conducted in strict accordance with the guidelines and regulations set forth by the Institute of Marine Biology FEB RAS (IMB FEB RAS), Animal Resources Center and Institutional Animal Care and Use Committee. The protocol was approved by the Committee on the Ethics of Animal Experiments of the IMB. All masu salmon manipulations were performed under solution of tricaine methanesulfonate (MS-222, Sigma) anesthesia, perfused with $\mathrm{PB},(\mathrm{pH} 7.4)$, and perfusion fixed with 4\% PFA, in PBS.

\subsection{Morphological Study of the Cerebellum}

An intracranial cavity of immobilized animal was perfused via the syringe with the 4\% PFA, in PBS (pH 7.4). After perfusion in PBS the brain of fishes was removed from the cranial cavity and fixed in the same solution for two hours at $4^{\circ} \mathrm{C}$. The brain was cryoprotected for two days in $30 \%$ sucrose solution at $4{ }^{\circ} \mathrm{C}$. Serial frontal sections of $O$. masou brain (thickness $50 \mu \mathrm{m}$ ) were produced on a freezing microtome Cryo-Star HM 560 MV (Germany). Sections were mounted on gelatinized slides and dried at room temperature. Morphological identification of the brain neurons were conducted by staining with a water solution of methyl green within 15 min according to the technique of Brasher $[8,9]$. Slides were washed with distilled water during 10 seconds and then differentiated for $10 \mathrm{sec}$ in $96 \%$ ethanol. The preparations obtained were dewatered using a conventional technique and embedded in medium BioOptica (Italy).

\subsection{Experimental Injury of Cerebellum}

A sterile 20-gauge needle was inserted into the cerebellum [corresponding to 10] of $O$. masou to create a 1-mm-deep stab wound in corpus cerebellum with the subsequent injection of carbocyanine dye DiI (1,1'-dioctadecyl-3,3,3'3'tetramethylindocarbocyanine perchlorate (Aldrich, Sigma, USA) in the damaged zone. The area of a trauma extended both on the dorsal molecular and the granular layer of corpus cerebellum and does not affect other parts of the brain. Immediately after creating the lesion, the animals were put into fresh water to recover. During the first hour after injury the animals showed increased locomotor activity (jumped out of the water and made rotary motions). These changes in motor activity of fish reveal a damage of cerebellum structure.

\subsection{Identification of Apoptosis by Detecting Fragments of DNA (TUNEL Labeling)}

To reveal apoptotic cells in the cerebellum of juvenils $O$. masou, we used a technique for immunoperoxidase labeling of fragmented DNA chains, called Terminal deoxynucleotidyl transferase dUTP nick end labeling (TUNEL). After 2-h-long fixation in $4 \%$ solution of PFA on 0.1 M PBS ( $\mathrm{pH} 7.2$ ), dissected brain was washed out for $24 \mathrm{~h}$ in $0.1 \mathrm{M}$ PBS. Then, these samples were put in $30 \%$ solution of sucrose based on phosphate buffer $(0.1 \mathrm{M})$ for cryoprotection. Frontal slices (50 $\mu \mathrm{m}$ thick) were prepared using a freezing microtome (Cryo-Star HM 560 MV). To identify TUNEL-positive structures, we used an immunoperoxidase identification system, ApopTag Peroxidase In Situ Apoptosis Detection Kit (Chemicon International Inc., USA). For blocking endogenous peroxidase, the slices were incubated in $1 \%$ solution of hydrogen peroxide for $3 \mathrm{~min}$ and then washed out two times for $5 \mathrm{~min}$ in phosphate buffer. The slices were covered with a smoothing buffer $(75 \mu \mathrm{l})$ and kept for $10 \mathrm{sec}$ at room temperature. Then, the slices were slightly dried, subjected to the action of TdT enzyme $\left(55 \mu \mathrm{l} / 5 \mathrm{~cm}^{2}\right)$, incubated in a humid chamber for $1 \mathrm{~h}$ at $37^{\circ} \mathrm{C}$, and immersed in a stop buffer for $10 \mathrm{~min}$. The slices were washed out in PBS at room temperature (three times for $1 \mathrm{~min}$ with changing of the solution), again dried, covered with antidioxygenin conjugate $\left(65 \mu 1 / 5 \mathrm{~cm}^{2}\right)$, and incubated in a humid chamber for $30 \mathrm{~min}$. To detect the reaction products, cerebral slices were incubated in the substrate for identification of peroxidase (VIP Substrate Kit; Vector Labs, USA) with control of the development of color under a microscope, washed out in three changes of PBS, and mounted on glass slides. The cell nuclei were subjected to final staining with methyl green according to the technique of Brasher [8]. The preparations obtained were dewatered using a conventional technique and embedded in medium BioOptica (Italy).

\subsection{Microscopy}

For morphological and morphometric analysis the motorized inverted microscope Axiovert Apotom $200 \mathrm{M}$, 
(Carl Zeiss, Germany) was used. On the basis of morphometric analysis we allocated 4 morphological types of cells. Morphometric parameters and studied correlations between some of the parameters were analyzed by Microsoft Excel 2010.

\subsection{Statistical Analysis}

For statistical and morphometric analysis we used software of microscope research class Axiovert Apotom 200 M, (Carl Zeiss, Germany). Data are expressed as the mean \pm S.E.M. and are analyzed with «Descriptive Statistics» program for Microsoft Excel 2007. Density of distribution of the cells was evaluated by methods of variation statistics. P-values $<0.05$ were considered to be statistically significant.

Apoptotic index was calculated with using software Axiovision Axiovert $200 \mathrm{M}$ at objective $40 \mathrm{x}$ in five, incidentally chosen fields of vision for each area. The apoptotic index was calculated to per field area, corresponding to $10121.7 \mu^{2}$.

$$
\text { apoptosis index }=\frac{\mathrm{n} \text { TUNEL- } \mathrm{n} \text { positive nuclei }}{\text { total } \mathrm{n} \text { nucleis }} * 100 \%
$$

\section{Results}

\subsection{Characteristic Changes in Cellular Structure in Injury Zone of Cerebellum}

The damaging effects (mechanical injury) have been applied on the dorsal surface of corpus cerebellum of $O$. masou. The zone of trauma extended from the dorsal molecular layer to deep of granular layer (Fig. 1A). The total length of the puncture was $1205.71 \mu \mathrm{m}$. For the characteristic of the histological changes in area of injury the microscopic analysis was performed.

Two days after injury in the cerebellum of $O$. masou changes of cellular structure in molecular and in granular layers were identified. The most characteristic changes include extensive cell migration from the area of regional neurogenic niches and the largest zone of secondary neurogenesis, located in the dorso-medial part of corpus cerebellum (Fig. 1B). In the granular layer, increased cell density was observed at the distance of about $250 \mu \mathrm{m}$. In this area, a high concentration of the cell bodies was found. We assume that most part of the cells migrated from regional neurogenic niches and dorso-medial region. The highest cell density was detected directly nearby the puncture area and gradually decreased with a far from the area of injury. Near the area of injury, TUNEL-labeling components, representing different stages of the apoptotic process were diagnosed (Fig. 1B). So, we identified dense apoptotic bodies representing the final stage of nuclear degradation, coarse chromatin condensation and apoptotic cell degranulation. The average size of apoptotic cells was 8-10 $\mu \mathrm{m}$. In zones of localization some apoptotic fragments were present in large cells with basophilic cytoplasm, the size of their bodies is about $13 \mu \mathrm{m}$. These cells usually have the irregular shape and cytoplasmic outgrowths. Presumably, these cells correspond to regional microglia/macrophages involved in phagocytosis and disposal of apoptotic fragments. Along with individual apoptotic elements were observed small conglomerates, including up to 3 apoptotic cells. The area of injury passed through the granular layer, where the cell density is very high, which explains the relatively low value of the apoptotic index unlike neurogenesis zones (Fig. 1B). Another variety of apoptotic bodies were small TUNEL- labeling elements representing degranulate fragments of damaged cells. An apoptosis index in area near the injury was $0.7 \%$.

In the molecular layer was a significant increase in small lacking signs of morphological differentiation of cells in the most superficial layer. In the thickness of the molecular layer longitudinal rows of elongated cells directed from the surface layer to the area of injury were often observed (Figure 1B). The number of cells on a profile field (at magnification 40x) was 237. These changes in cell morphology of the surface layer indicate the processes of cell migration from the surface region in the area of injury. In the surface layer with a high density of cells were identified small TUNEL-labeling elements corresponding to products degranulation of cells in the area. Two types of cells have been allocated: rod-shaped cells with body from 10 to $21 \mu \mathrm{m}$ and cells with a length of elongated body from 7 to $10 \mu \mathrm{m}$ (Table).

\subsection{Lateral and Dorsal-Medial Part of the Molecular Layer of Corpus Cerebellum}

After applying injury to the cerebellum we found changes in the morphological structure in various areas of molecular layers: the dorsal-medial and lateral. Histological structure of the molecular layer stained with methyl green according to Brashe has been studied (Fig. 1B). There was a significant increase in the number of undifferentiated cells in zones of secondary neurogenesis. The average number of cells in this area after the damaging effects was 251 cells per field (at magnification 10x). Besides the morphological structure of these areas of a molecular layer (dorso-medial and lateral), apoptosis in this area has been diagnosed (Table). The number of cells in the surface area and thickness of the molecular layer significantly increased. The main cell types in this area and their morphological characteristics are given in the table. The average number of cells per field in a molecular layer was 271 . The majority of cells had an elongated shape and possibly was in a condition of migration. In addition, there was a significant increase in the number of undifferentiated cells of the granular layer. The number of cells in this area was 400 per $31277 \mu \mathrm{m}^{2}$ (Fig. 2). In the surface layer, the patterns of typical tangential migration from areas of proliferation aimed at the injured area were revealed. In addition to the tangential migration were revealed multiple patterns of a radial migration from the area of secondary neurogenesis (a dorso-medial matrix zone of a cerebellum) to injury site. At such option of migration, the cells were directed perpendicular to the surface layer (Fig. 1B).

In the dorso-medial and lateral zones of molecular layer cases of apoptosis were diagnosed. Most of TUNEL-labeling elements were detected in the granular layer (Fig. 1B). Most of these elements were apoptotic bodies of about $10 \mu \mathrm{m}$, found in 
the dorso-medial part of the corpus cerebellum, adjacent to a zone of secondary neurogenesis. It is very probable that the secondary matrix zone is located in the molecular layer near the region of localization of apoptotic cells. We believe that the existence of apoptotic cells in the granular layer is associated with increased activity of the secondary matrix zone. Another area of localization of apoptotic bodies is a boundary between molecular and granular layer (Fig. 1C). Apoptotic bodies were also observed in the thickness of molecular layer. Here we identified both fragments of cell's degranulation and terminal apoptotic bodies. Average apoptosis index in the molecular layer was $5 \%$.
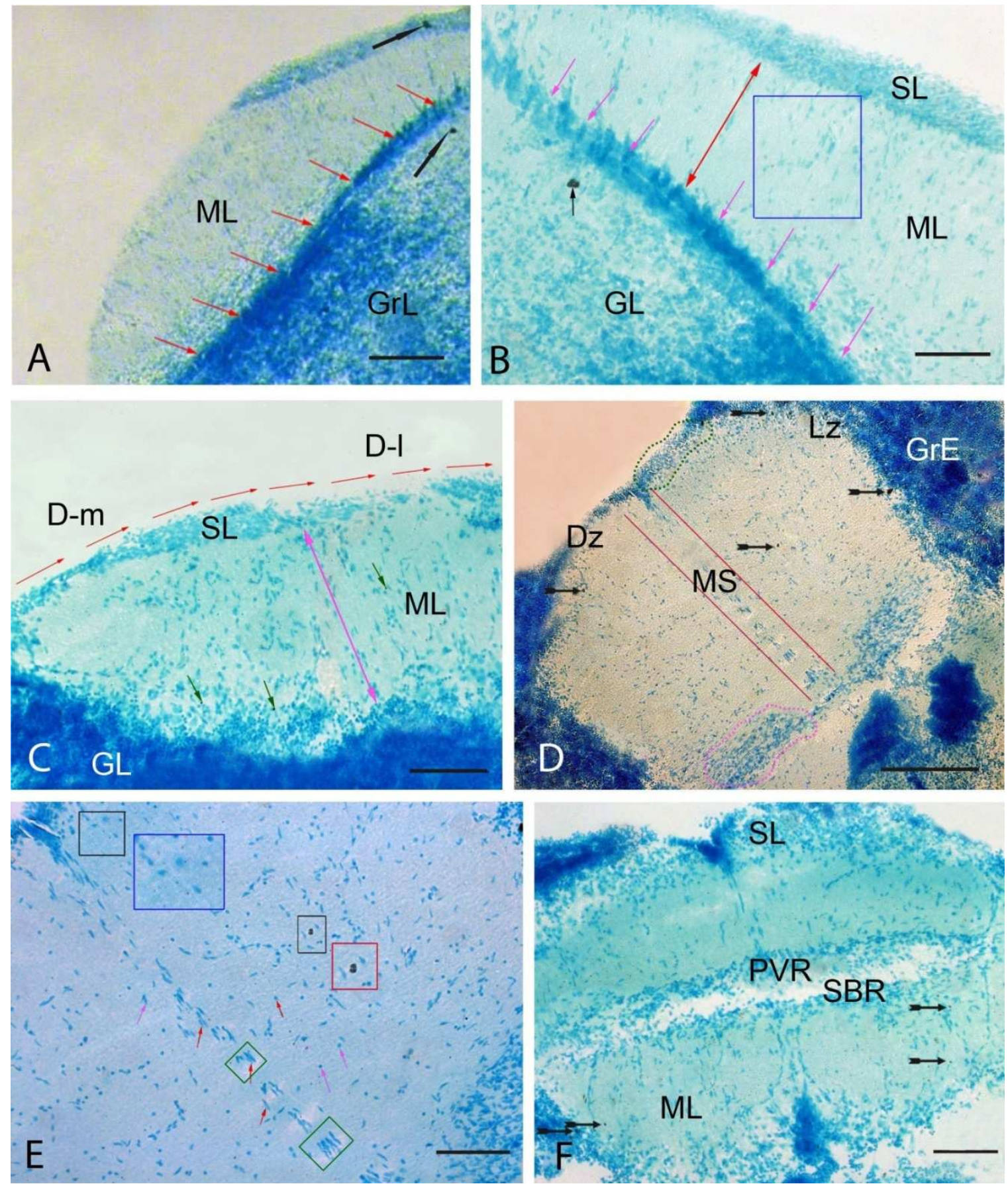

Figure 1. Patterns of cell migration and apoptosis in the cerebellum of juvenile masu salmon Oncorhynchus masou after injury. A - common view the areas of trauma, red arrow shows the area of puncture, black arrows (here and below) shows apoptotic bodies, $\boldsymbol{B}$ - dorso-medial part of corpus cerebellum, which contains migrating from the surface layer (SL) cells (contoured by rectangle), the red arrow indicates the direction of radial migration; $C$ - lateral part of the molecular layer (ML) contained cells migrating to the area of injury, red arrows show the direction of the tangential migration, pink arrow - radial migration, green arrows show TUNEL-labeling fragments of degranulated cells, D-m - dorso-medial, D-l - dorso-lateral area; $\boldsymbol{D}$ - caudal part of cerebellar body, green contour plot is limited dorsal zone (DZ), pink contour plot is limited basal zone, median suture zone (MS) is between the red lines, LZ - lateral zone, GE granular eminences; $\boldsymbol{E}$ - the area median suture, green squares delineated clusters of migrating cells, in red and blue squares are small TUNEL-labeled fragments (in blue), and apoptotic cells (in red), pink and red arrows show the different types of cells; $\boldsymbol{F}$ - Valvula cerebelli; PVR - periventricular region, SBR - subventricular region, $M L$ - molecular layer, GL - Granular layer. Scale bar: A, F - $200 \mu \mathrm{m}, B-E-100 \mu \mathrm{m}$. 
Table. Morphological and morphometric parameters of cells $(M \pm m)$ and frequency of apoptosis in the cerebellum of juvenile Oncorhynchus masou after injury

\begin{tabular}{|c|c|c|c|c|c|}
\hline \multirow{2}{*}{ Areas of the cerebellum } & \multicolumn{3}{|c|}{ Cell types in the cerebellar zones } & \multirow{2}{*}{$\begin{array}{l}\text { Proportion of cells, } \\
(\%)\end{array}$} & \multirow{2}{*}{$\begin{array}{l}\text { Total proportion of } \\
\text { apoptotic bodies, }(\%)\end{array}$} \\
\hline & Cell types & Large diameter, $(\mu \mathrm{m})$ & Small diameter, $(\mu \mathrm{m})$ & & \\
\hline \multirow{4}{*}{ Molecular layer } & 1 & $5,04 \pm 0,51$ & $3,93 \pm 0,69$ & 2 & \multirow{4}{*}{5} \\
\hline & 2 & $6,26 \pm 0,53$ & $5,25 \pm 0,53$ & 8,5 & \\
\hline & 3 & $9,51 \pm 0,41$ & $3,53 \pm 0,63$ & 55 & \\
\hline & 4 & $13,63 \pm 2,33$ & $4,25 \pm 0,50$ & 34,5 & \\
\hline \multirow{2}{*}{ Granular eminences } & 1 & $5,21 \pm 0,38$ & $4,71 \pm 0,42$ & 85 & \multirow{2}{*}{2} \\
\hline & 2 & $7,19 \pm 1,33$ & $4,30 \pm 0,42$ & 15 & \\
\hline \multirow{4}{*}{ Median suture } & 1 & $5,79 \pm 0,13$ & $3,58 \pm 0,12$ & 23 & \multirow{4}{*}{8} \\
\hline & 2 & $8,76 \pm 0,34$ & $3,07 \pm 0,32$ & 15 & \\
\hline & 3 & $9,78 \pm 0,18$ & $2,76 \pm 0,15$ & 17,50 & \\
\hline & 4 & $22,88 \pm 0,58$ & $2,11 \pm 0,17$ & 44,50 & \\
\hline \multirow{4}{*}{ Dorsal part } & 1 & $5,26 \pm 0,47$ & $4,03 \pm 0,66$ & 53 & \multirow{4}{*}{4} \\
\hline & 2 & $6,26 \pm 0,27$ & $5,03 \pm 0,60$ & 12 & \\
\hline & 3 & $8,78 \pm 0,46$ & $3,89 \pm 0,46$ & 12 & \\
\hline & 4 & $11,15 \pm 0,84$ & $3,83 \pm 0,65$ & 23 & \\
\hline \multirow{4}{*}{ Valvula cerebelli } & 1 & $5,52 \pm 0,34$ & $4,74 \pm 0,60$ & 36 & \multirow{4}{*}{5} \\
\hline & 2 & $6,77 \pm 0,58$ & $5,18 \pm 0,70$ & 34 & \\
\hline & 3 & $9,13 \pm 0,65$ & $3,16 \pm 0,48$ & 19 & \\
\hline & 4 & $12,60 \pm 1,88$ & $3,45 \pm 1,13$ & 11 & \\
\hline
\end{tabular}

\subsection{Characteristic of the Caudal Part of Corpus Cerebellum}

Another area with significant histological changes after traumatic injury of cerebellum was the caudal part of corpus cerebellum. As part of this area were analyzed dorsal part, zone of granular eminences located laterally and area of median suture (Fig. 1D). Major part of caudal part of corpus cerebellum was represented by molecular layer. In the dorsal area of caudal part of corpus cerebellum we also observed a significant increase of cell number compared with the intact brain. Characteristics of the most superficial layers were similar with previously described structure of the surface area of molecular layer of corpus cerebellum. The heterogeneous populations containing four types of cells were revealed in the deeper layer. Morphometric characteristics of all cell types are presented in the Table. Cells differed in morphological parameters. The first type is represented by small round cell size from 4 to $6 \mu \mathrm{m}$. These cells were the prevailing cellular type and accounted for $85 \%$ of all cells in the zone of granular eminences. The second type presented larger elements with diameter from 6 to $10 \mu \mathrm{m}$. Such cells were presented in smaller quantity $-15 \%$, and represent more differentiated and possibly later stages of development of cells of 1st type. In the zone of the median seam we noted a large concentration of cells belonging to different types. The sizes of cells of the migrating population in a zone of the central seam are: from $8.1 \mu \mathrm{m}$ to $18.75 \mu \mathrm{m}$, the number of such cells was 59. The total number of cells per field (at magnification 20x) was 265 , of which the dominating cells were migrating cells of the $3 \mathrm{rd}$ and 4 th types. The sizes of the cells of 3rd type from 7 to 10 microns, they accounted for $17 \%$ (Fig. 1D). Cells of $4^{\text {th }}$ type have a size from 10 to 21 microns constituting $45 \%$ of the total number (Fig. 3). The number of migrating cells was 165 per field. The total number of cells of the 3rd and 4th types was $62 \%$, while the percentage of proliferating cells were (with a large diameter of $10 \mu \mathrm{m}$ ), $38 \%$ of the total. The average apoptosis index in the molecular layer was $8 \%$.

\subsection{Granular Eminences}

The zone of granular eminences represents the area of the maximum cellular density and is traditionally considered, as area of embryonic neurogenesis of fishes [10]. In our studies, high morphogenetic activity, identified by morphological parameters, was established for the study of development of juvenile $O$. masou. In laterally spaced granular eminences (Fig. 1D), we revealed two morphological classes of cells (see table). The predominant cells with a body length of up to $6 \mu \mathrm{m}-85 \%$ were of 1 st type; the 2 nd type of cells with a body length of up to $10 \mu \mathrm{m}$ was $15 \%$. When compared with other areas of the cerebellum, containing to 4th types of cells in granular eminences were identified only two cell types. This may indicate that this region is an area in which neurogenesis processes prevail, and migration processes are expressed to a lesser extent. In this zone, has also been identified TUNEL-labeling fragments larger condensed and collected in a single group (Fig. 1D, Table). Apoptotic index zone granular eminences was - $2 \%$ in dorsal $-4 \%$ and in the lateral $-1 \%$.

\subsection{Median Seam}

In a zone of a median seam was noted a large concentration of cells belonging to different types. The size of cells of migrating population in the zone of the central seam was from $8.1 \mu \mathrm{m}$ to $18.75 \mu \mathrm{m}$, the number was 59 . The total number of cells per field (at magnification 20x) is 265, which are dominated by migratory cells of the 3rd and 4th types (Table). The sizes of the cell bodies of 3rd type were from 7 to $10 \mu \mathrm{m}$, they accounted for $17 \%$ (Figure 1D). Cells 
of type 4 (from 10 to $21 \mu \mathrm{m}$ ) constitute $45 \%$ of the total number (Table). The number of migrating cells was 165 per field. The total number of cells of the 3rd and 4th types was $62 \%$, while the share of proliferating (with a large diameter of $10 \mu \mathrm{m}$ ), cells was $38 \%$ of the total. Average apoptosis index in the molecular layer was $8 \%$.

\subsection{Dorsal Zone}

The dorsal zone is the external area of the corpus cerebellum, adjacent to brain covers and located on the dorsal side (Fig. 1D). In this zone, have been identified type 4 cell whose morphological parameters are revealed in the table. Among the selected cell types were identified elements of $1 \mathrm{st}$ and 2nd types, based on morphological parameters corresponding proliferating population. The proportion of these cells was $65 \%$. Another population of cells represented elongated rod-like elements of 3rd and 4th types and having various orientations in space corresponding to tangential patterns of cell migration. The proportion of such cells was $35 \%$. Thus, dorsal zone is a combined field in which based on morphological criteria can identify proliferative and migratory cell populations. We identified TUNEL-positive elements; the quantity of such elements in the per field was 5 .

\subsection{Valvula Cerebelli}

$\mathrm{VC}$ is an evolutionarily unique area and is the most variable part of the cerebellum of fishes in comparison to other parts of brain. The most part of $\mathrm{VC}$ is placed under the optic tectum hemispheres inside the tectal ventricle. Valvula is the unpaired part of fish's cerebellum and belongs to the youngest brain structures from the point of view of a phylohistogenesis [11]. In the valvula of $O$. masou and other species of fish were observed active proliferative processes, both during embryogenesis and later in adult stage of ontogenesis. In the valvula intensity of proliferative processes during the post-embryonic period is the highest to compare with other brain structures of fish. Thus, the valvula cerebelli is the active center of neurogenesis in young and adult animals. In valvula of juvenile $O$. masou we identified several zones: periventricular one surrounding cerebellar aqueduct adjacent to subventricular region, the molecular layer and the surface layer (Fig. 1E). In the periventricular region the concentration of cells repeatedly exceeded to that in other parts of the valvula. In the valvula have been identified all four major types of cells (Table). In this zone predominated the undifferentiated cells of 2nd type (36\%), and cells of 1 st type (34\%). Both of them contain $70 \%$. The migrating cells (the 3rd and 4th types) were also present, but in smaller number (30\%). Apoptosis index in the molecular layer is $5 \%$.

\section{Discussion}

Animals have been in the active growth phase, which also reflects on the state of morphogenetic areas of the brain. This is due to the increased proliferative activity of cells in morphogenetic zones of the cerebellum and in periventricular regions of the brain. In studies on Danio rerio it was found that after injury of cerebellum proliferative activity of cells was observed in the outer regions of brain, adjacent to brain covers, and in valvula cerebelli [10] and in the granular eminences. These data were obtained by experimental marking of a BrdU [1, 2] and labeling with some markers of cell proliferation [11]. High level of neurogenesis after traumatic injury was identified in the cerebellum of Apteronotus leptorhynchus [12]. This animal belonging to the group of highly specialized electrical gimnotiform fish; cerebellum of $A$. leptorhynchus takes up $75 \%$ of brain volume is the largest neurogenetic center during all fish's life, as in conditions of traumatic influence [12].

A research was carried out on individuals of juvenile $O$. masou, in which the brain is in a state of indeterminate growth. There was an increased level of proliferative activity. In such conditions, it is necessary to take into account the background level of activity of proliferative zones associated with the implementation of morphogenetic programs of a brain growth. Thus, in their observations and conclusions, we relied on data from the literature describing the state of the matrix zones of the cerebellum in adult fish, and also change of a condition of these zones when drawing the damaging influence $[1,2,5,7]$.

In juvenile $O$. masou has a high initial level of proliferation in the primary matrix zone of the brain (periventricular matrix zone) and proliferative activity in the zones, which have been identified in adult animals [13, 14]. These data are diagnosed by immunolabeling of a proliferative cell nuclear antigen (PCNA), and using morphological methods to assess the number of mitoses in the matrix areas of brain. This area of the brain in the cerebellum is the dorso-medial region. Proliferation of cell was described in adult Danio rerio [2] and A. leptorhynchus [12]. In our study after damaging impact to the cerebellum high proliferative activity has been identified in the dorsomedial zone and granular eminences of corpus cerebellum and in vestibulo-lateral lobes. Thus, it is possible to conclude that the activity in dorso-medial zone of cerebellum, and in granular eminences can be a consequence of background morphogenetic and genetic activity of the fish brain, which increases with the damaging effects.

Existence of newly produced cells in the superficial layer of the cerebellum, is also partly associated with a morphogenetic activity of the brain, and with damaging influence too. As a result of the damaging impact of the cerebellum in juvenile $O$. masou was revealed induction of proliferative activity in both zones of adult neurogenesis and in the surface layer. The cumulative performance of proliferative activity of these zones in juvenile $O$. masou is higher than described activity of BrdU in adult Danio rerio $[2,5,15]$. At the sections of a corpus cerebellum of adult Danio rerio no more than 5-10 cells on per field were identified [15]. This may be due to the methodological aspects (the use of thin sections of the brain) and with the physiological status of animal. In adult animals the frequency 
of proliferative processes identified by BrdU labeling, is also low, because the BrdU labeling is taken into account for a limited period of time (1-2 hours) for BrdU incorporation.

In studies of the proliferative activity in the tectum at the 3 month-old juvenile trout [11] the presence of small amounts of PCNA-immunopositive cells per field has been demonstrated. Nevertheless, we tend to assume a low level of antibody immunoreactivity used in the above mentioned study; as the results of studies in brain of $O$. masou showed a higher proliferative activity [14]. The results of morphological observation in the nuclei of cells stained with methyl green show that in the surface layer of the cerebellum indicates the presence of a large number of undifferentiated cells with high nuclear-cytoplasmic relations. Such cells based on morphological criteria can be referred as the proliferating cells (1st and 2nd types) and cells to an initial stage of differentiation and/or migration (3rd and 4th types, see the table).

Thus, the proliferative response in cerebellum of juvenile O. masou formed with respect to the background level of proliferative activity of matrix zones of brain. Activity of regional neurogenic niches as a result of the damaging influence supplements an overall picture. The process of apoptotic cell death is a physiological response of the CNS of O. masou after damaging impact on the cerebellum. However it is necessary to consider that the phenomenon of apoptosis is characteristic also for morphogenetic processes during a post-embryonic development of a brain of fishes [16, 17].

So the phenomenon of apoptosis in normal brain development of fishes has been described in A. leptorhynchus $[1,10]$. It was found that as a result of the proliferative activity of matrix zones of a cerebellum formed cells with somatic aneuploidy. Such material is defective from the point of view of diploidic quantity of chromosomes and usually it gets eliminated via apoptosis [16]. In study of adult individuals of the Amur sturgeon, existence of high apoptotic indices in different parts of the central nervous system, including integrative centers (optic tectum, cerebellum) and sensory centers (nuclei of V, VII and VII craniocereberal nerves) of a medulla oblongata was revealed [17]. These properties are characteristic of the proceeding morphogenetic activity of different brain centers, which continue to grow throughout the animal's life. Arising de novo cells may be incorporated into the existing neural network, not only during embryonic neurogenesis but also in adult animals too [5]. This phenomenon is characteristic for development of the sensory areas of brain updated with new structural elements as the animal grows. Apoptosis in such physiologically active developing systems can play a role of the physiological filter regulating the emergence of new cells.

It has been hypothesized that in vertebrates with indeterminate growth, such as teleosts, the primary function of adult neurogenesis is to ensure a constant ratio of the number of central neuronal elements within motor/sensory pathways relative to the number of peripheral elements muscle fibers and receptor cells - when the number of peripheral elements grows [5]. Such a function is consistent with the existence of an enormous neurogenic potential in the teleostean brain, which correlates with continuous peripheral growth and the constant increase in the number of individual muscle fibers or receptor cells [18]. By contrast, growth in mammals is primarily the result of an increase in the size, not in the number, of individual peripheral elements [19]. As a corollary, the matching hypothesis $[1,5]$ predicts that, when the mode of muscle growth is shifted from hyperplasia to hypertrophy in the course of the evolution of mammals, the neurogenic potential of CNS structures forming part of the motor pathway was reduced in parallel. Similarly, the number of neurogenic regions and teleost fish as a model system the rate of neurogenesis decreased in brain areas associated with sensory processing when the continuous formation of new sensory cells in the sensory organs during adulthood was gradually abandoned.

In our work related to the study of apoptosis in the cerebellum of injured juvenile $O$. masou were studied cells by labeling of DNA fragments (130 base pairs). The existence of such DNA fragments is characteristic of the later stages of apoptosis. In the zones surrounding the area of injury were identified single TUNEL-labeling elements and clusters consisting of several elements. The greatest number of TUNEL-labeling structures was detected in areas adjacent to morphogenetic zones of a cerebellum. The area of trauma and the territory surrounding injury area was another zone where concentration of TUNEL-labeling structures considerably exceeded the background level.

Large TUNEL-labeling bodies represent the final stage of degradation of apoptotic cells. Such large condensed fragments cannot be utilized by macrophages/microglia [6]. Similar observations were also identified in morphogenetic study on mammals [20, 21]. Other version of TUNELlabeling, when the marked elements are the small, poorly condensed particles representing products of degranulation of cages, eliminable through apoptosis [21]. In such cases, the "cell debris" is identified in the big territories located in various parts of a cerebellum. Similar patterns of distribution of TUNEL-labeling structures in the cerebellum of $O$. masou were found everywhere. Different apoptosis indices in various parts of a cerebellum indicate various intensity of apoptosis in matrix zones, areas of trauma and in intact areas of $O$. masou cerebellum after injury (Fig. 2).

We investigated the cellular response in various anatomic zones of the cerebellum of juvenile $O$. masou: dorsal and lateral parts of the corpus cerebellum, granular eminences, the molecular layer of the valvula cerebelli and the caudal part of corpus cerebellum. Traumatic influence was applied to the corpus cerebellum on the border between the molecular and granular layers. As a result of TUNELlabeling, we established that the intensity of the different parts of apoptosis is not the same (Fig. 2). However, it is necessary to take into account the considerable density of distribution of cell bodies in a granular layer. In all vertebrates, the granular layer of the cerebellum is a zone of maximal density localization of cell bodies forming somatosomatic contacts. In the granular layer of the cerebellum of 
juvenile $O$. masou density distribution of cells was also very high, which affected the final value of the apoptotic index in the area of injury. Despite the presence of TUNEL-labeled elements in areas of injury, apoptosis index values were low (Fig. 2) that is connected inversely proportional to the density distribution of the cells in this zone. The highest value of the index of apoptosis was detected in the molecular layer. This is due to the fact that the molecular layer is the main zone of radial migration of cells formed as a result of the reparative neurogenesis and natural morphogenetic processes. It was found that the maximal number of apoptotic cells observed in the areas of cell migration and this suggests that some of the cells are eliminated in the process of radial migration. Another high rate of apoptosis index was detected in the dorsal zone of corpus cerebellum; this area is corresponding to zone of secondary neurogenesis (Fig. 2). For example, in a matrix zone of the brain intensity apoptotic processes is sufficiently high as compared with other regions. This testifies to elimination of newly formed cells at the stage of their localization in the matrix zones. In a zone of granular eminences, apoptotic index was only $2 \%$, which is also associated with a high density of the cell distribution. Another factor affecting the value of the apoptotic index was the fact that the zone of granular eminences - the zone of embryonic neurogenesis, which may takes less involved in reparative neurogenesis than the dorsal zone.

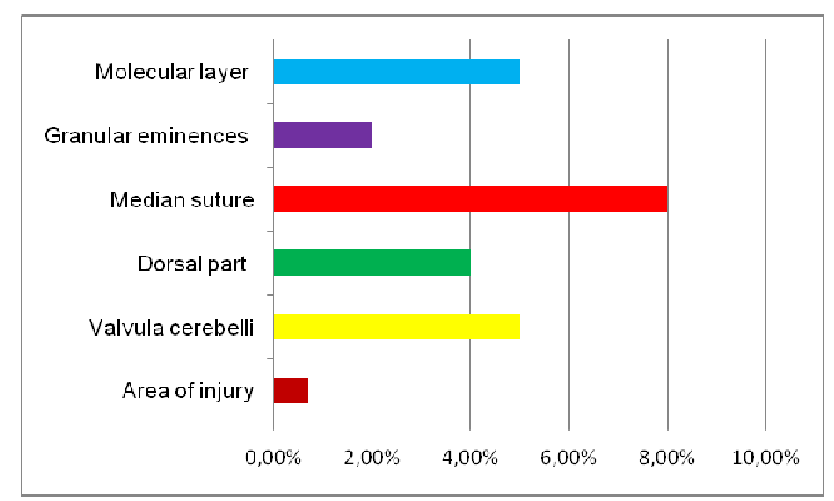

Figure 2. The intensity of apoptosis in the cerebellum of juvenile Oncorhynchus masou after injury. At the $x$ axis is shown the apoptotic index (\%); at the $y$ axis is shown areas of cerebellum.

This indicates that the intensity of the physiological elimination of cells varies in regions dominated by background morphogenetic processes and physiological repair processes following traumatic influence, and in areas distant from those zones. In A. leptorhynchus studies it was found that the intensity of apoptosis varies at different times. Thus, the most intense elimination of cells observed in the first hours after the application of the damaging influence. This process weakens over time and stops completely to $21 \mathrm{st}$ days after injury to the adult animal [5]. In our study on the second day after injury was not revealed an intense pattern of TUNEL-labeled structures. This may be due to the fact that the most active apoptosis in the cerebellum of $O$. masou after injury occurs in the earliest period. Considering the high intensity of proliferation in the cerebellum of juvenile $O$. masou this assumption seems reasonable. Nevertheless, apoptosis was recorded in the zone adjacent to the area of injury, which certainly indicates the presence of the recycling mechanism in damaged cells. During this period of time (2 days after injury) in the zone of damage has already been revealed increased density of undifferentiated cells. This demonstrates later stages of the repair process. We believe that reparative processes of neurogenesis in juvenile $O$. masou pass in earlier date compared to the currently available literature data $[1,5]$. This is due to more intensive background of morphogenetic activity in the cerebellum of $O$. masou. It is explained by existence of more intensive background morphogenetic activity in a cerebellum of juvenile $O$. masou compared with adult animals.

The conducted researches allow us to conclude that after the application of the damaging influence in the field of injury, patterns of tangential and radial cell migration are observed. The greatest zone of cell migration is represented in the dorsal part of corpus cerebellum, and also in zones of secondary neurogenesis. After traumatic injury of cerebellum of $O$. masou was identified four types of cells, presented proliferating populations (1st and 2nd types) or populations which are in the early stages of differentiation and/or migration (3rd and 4th types, see table). As a result of traumatic injury of cerebellum of juvenile $O$. masou, we identified two groups of TUNEL-labeling structures: large TUNEL-labeling bodies represent the final stage of degradation of apoptotic cells and small weakly condensed particles - products of degranulation of cells eliminable via apoptosis.

In matrix zones, areas of a trauma and in intact areas of the cerebellum of juvenile $O$. masou were identified different levels of apoptotic activity: (a) The highest value of the index of apoptosis in the cerebellum traumatic impact was detected in the molecular layer (5\%), which is the main area of the radial cell migration; (b) In the zone of injury for 2 days was detected maximum cell density, that determines the lowest value of the apoptotic index $(0.7 \%)$ in this region; c) The dorsal zone has the highest apoptosis index (4\%), and takes a more active part in the processes of reparative neurogenesis compared with other areas. The intensity of the apoptosis varies in areas dominated by background morphogenetic processes and physiological processes of repair after traumatic influence, as well as in areas remote from these zones.

The existence of such cell-cell interaction in different stages of animal ontogenesis has important implications from a biomedical point of view because then it should be possible to reactivate these elements under certain environmental conditions by turning off or on the switches that control them [21]. Indeed, quiescent stem cells have been discovered in the adult mammalian brain that can be activated by certain signals from the cellular environment, such as epidermal growth factor [22]. Clearly, these adult stem cells could provide the substrate for the development of a cell replacement therapy based on the intrinsic ancestral potential of the adult human brain. The study of regeneration- 
competent organisms could play an important role in identifying the factors required to reactivate this potential.

This work was supported by Program of fundamental investigation FEB RAS «Far East» 2015-2017 (grant № 15-I6-116); Grant of President of Russian Federation (MD4318.2015.4) and DST-INSPIRE Faculty Grant (IFA14LSBM-104), from the Department of Science and Technology (DST), Government of India.

\section{References}

[1] Zupanc G.K. (2006). Neurogenesis and neuronal regeneration in the adult fish brain. J. Comp. Physiol., 192:649-670.

[2] Zupanc G.K., Wellbrock U.M., Sîrbulescu R.F., Rajendran R.S (2009). Generation, long-term persistence, and neuronal differentiation of cells with nuclear aberrations in the adult zebrafish brain. Neuroscience, 159:1338-1348.

[3] Williams R.W. (2000). Mapping genes that modulate brain development: a quantitative genetic approach. Results Probl. Cell Differ., 30:21-49.

[4] Takeda A., Nakano M., Goris R.C., Funakoshi K. (2008). Adult neurogenesis with 5-HT expression in lesioned goldfish spinal cord. Neuroscience, 151:1132-1141.

[5] Zupanc G.K., Sîrbulescu R.F. (2013). Teleost fish as a model system to study successful regeneration of the central nervous system. Curr. Top. Microbiol. Immunol., 367:193-233.

[6] Zupanc G.K., Clint S.C., Takimoto N., Hughes A.T., Wellbrock U.M., Meissner D. (2003). Spatio-temporal distribution of microglia/macrophages during regeneration in the cerebellum of adult teleost fish, Apteronotus leptorhynchus: a quantitative analysis. Brain Behav. Evol., 62:31-42.

[7] Pouwels E. (1978). On the development of the cerebellum of the trout, Salmo gairdneri: I. Patterns of cell migration. Anat. Embryol., 152:291-308.

[8] Merkulov A.G. (1969). Course of patologohistological techniques. Leningrad, Medicine, 423 p. (In Russian).

[9] Romeys B. (1953). Microscopic technique. Moscow. Foreign Literature Publishing House, 718 p. (In Russian).

[10] Zupanc G.K. (1999). Neurogenesis, cell death and regeneration in the adult gymnotiform brain. J. Exp. Biol., 202:1435-1446.
[11] Candal E., Anadon R., Bourrat F., Rodríguez-Moldes I. (2005).Cell proliferation in the developing and adult hindbrain and midbrain of trout and medaka (teleosts): a segmental approach. Brain Res. Dev. Brain Res., 160:157-175.

[12] Zupanc G.K., Ott R. (1999). Cell proliferation after lesions in the cerebellum of adult teleost fish: time course, origin, and type of new cells produced. Exp. Neurol., 160:78-87.

[13] Pushchina Ye.V., Obukhov D.K., Varaksin A.A. (2012). Neurochemical markers of cells of the periventricular brain area in the masu salmon Oncorhynchus masou (Salmonidae). Russian J. Develop. Biol., 43(1): 35-48

[14] Pushchina E.V., Obukhov D.K., Varaksin A.A. (2013). Features of adult neurogenesis and neurochemical signaling in the cherry salmon Oncorhynchus masou brain. Neural Regen. Res., 8:13-23.

[15] Ekström P., Johnsson C.M., Ohlin L.M. (2001). Ventricular proliferation zones in the brain of an adult teleost fish and their relation to neuromeres and migration (secondary matrix) zones. J. Comp. Neurol., 436:92-110.

[16] Rajendran R.S., Zupanc M.M., Lösche A., Westra J., Chun J., Zupanc G.K. (2007). Numerical chromosome variation and mitotic segregation defect in the adult brain of teleost fish. Dev. Neurobiol., 67:1334-1347.

[17] Pushchina E.V., Obukhov D.K. (2011). Processes of proliferation and apoptosis in the brain of the Amur sturgeon. Neurophysiol., 43(4):271-286.

[18] Rowlerson A., Veggetti A. (2001). Cellular mechanisms of post-embryonic muscle growth in aquaculture species. Muscle development and growth. Ed. I.A. Johnston. San Diego, Acad. Press, pp 103-140.

[19] Rowe R.W., Goldspink G. (1969). Muscle fibre growth in five different muscles in both sexes of mice. J. Anat. 104:519-530.

[20] Nagamoto-Combs K., McNeal D.W., Morecraft R.J., Combs C.K. (2007). Prolonged microgliosis in the rhesus monkey central nervous system after traumatic brain injury. J. Neurotrauma, 24:1719-1742.

[21] Kalinichenko S.G., Matveeva N.U. (2007). Morphological characteristics of apoptosis and its significance in neurogenesis. Morphology, 131:16-28.

[22] Kondo T., Raff M. (2000). Oligodendrocyte precursor cells reprogrammed to become multipotential CNS stem cells. Science, 289:1754-1757. 